\title{
Abstract: Landmark-Free Initialization of Multi-Modal Image Registration
}

\author{
Julia Rackerseder ${ }^{1}$, Maximilian Baust ${ }^{2}$, Rüdiger Göbl ${ }^{1}$, Nassir Navab $^{1,3}$, \\ Christoph Hennersperger ${ }^{1,4}$ \\ ${ }^{1}$ Technische Universität München, Munich, Germany \\ ${ }^{2}$ Konica Minolta Laboratory Europe, Munich, Germany \\ ${ }^{3}$ Johns Hopkins University, Baltimore, USA \\ ${ }^{4}$ Trinity College Dublin, Dublin, Ireland \\ julia.rackerseder@tum.de
}

To achieve convergence, nonlinear deformable image registration tasks of partial-view 3D ultrasound and MRI, as often seen in US guided interventions or retrospective studies thereof, need to be initialized. In clinical practice corresponding 3D landmarks are selected in both images. Performing this depends on the geometrical understanding of the targeted anatomy and the modalityspecific appearance and is thus prone to error. Therefore, in [1] we propose a novel landmark-free initialization procedure that is robust in terms of target area overlap (pixels where target area and US volume are superimposed before initialization) as well as image overlap (pixels where MRI and US are superimposed). The method only requires $\mathrm{N}$ low-resolution coarse segmentations as input, which in most cases can be obtained automatically or with minimal user interaction, such as few pre-labeled pixels. A euclidean distance transform is applied to these $\mathrm{N}$ label maps, creating a multi-class distance map for both images. This leads to a minimization problem, where these maps are registered by optimizing our proposed similarity measure via a modified gradient descent scheme, which prevents unstable behaviour. The proposed method was evaluated, showing a success rate of $100 \%$ for registration tasks with initial target area overlap over $10 \%$. It also converges for all cases with image overlap of $30 \%$ or more.

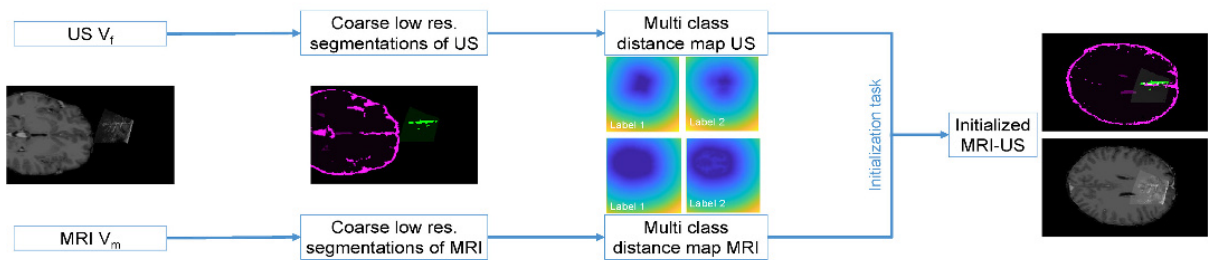

Fig. 1. Graphical overview of the method.

\section{References}

1. Rackerseder J, Baust M, Göbl R, et al. Initialize globally before acting locally: enabling landmark-free 3D US to MRI registration. Proc MICCAI. 2018; p. 827835. 